(2) Open Access Full Text Article

\title{
Reasons for reoperation after epilepsy surgery: a review based on a complex clinical case with three operations
}

This article was published in the following Dove Press journal:

Neuropsychiatric Disease and Treatment

5 July 2010

Number of times this article has been viewed

\author{
José F Téllez-Zenteno' \\ Farzad Moien-Afshari' \\ Lizbeth Hernández- \\ Ronquillo' \\ Robert Griebel ${ }^{2}$ \\ Venkat Sadanand ${ }^{2}$
}

'Department of Medicine, Division of Neurology, University of

Saskatchewan, Saskatoon, Canada;

${ }^{2}$ Department of Surgery, Division

of Neurosurgery, University of

Saskatchewan, Saskatoon, Canada
Correspondence: José F Téllez-Zenteno Department of Medicine, Division of Neurology Royal University Hospital, Saskatoon, SK, S7N 0W8 Canada Email jfthellez@yahoo.com

\begin{abstract}
The results of surgical treatment of epileptic seizures have gradually improved in the past decade, approaching $60 \%$ to $90 \%$ seizure-free outcome in temporal lobe epilepsy and $45 \%$ to $66 \%$ in extratemporal lobe epilepsy. Unfortunately some patients continue with seizures after epilepsy surgery and the studies have shown that approximately the $3 \%$ to $15 \%$ of patients with a previous failed surgical procedure are reoperated. Selected patients may be candidates for further surgery, potentially leading to a significant decrease in the frequency and severity of seizures. In patients with intractable partial epilepsy there are many possible factors, alone or in combination, that could be related to the failure of resection. Some of the factors could be genetic or acquired predisposition to epileptogenicity. In this article we report a case with intractable epilepsy that required three interventions to render seizure free. We analyzed our specific case in the light of previous reports on reoperation and enumerate the potential reasons for reoperation that could apply to all patients with failure of an initial procedure.
\end{abstract}

Keywords: intractable epilepsy, reoperation, epilepsy surgery, surgical failure

\section{Background}

The results of surgical treatment of epileptic seizures have improved in the past two decades, approaching $60 \%$ to $90 \%$ seizure-free outcome in patients with temporal lobe epilepsy and $40 \%$ to $60 \%$ in extratemporal lobe epilepsy. ${ }^{1}$ However, there are few alternative treatment options to patients who fail epilepsy surgery such as reoperation, use of electrical stimulation and new medications. Reoperation for recurrent temporal epilepsy was first reported in 1954 by Penfield. Selected patients may be candidates for reoperation, potentially leading to a significant decrease in seizure burden. The frequency of reoperation reported in the literature is variable, ranging from $3 \%$ to $14 \% .^{2-4}$

The success after a second surgical procedure is variable and different studies have reported different seizure-free rates. Awad et al reported that $47 \%$ of patients were seizure free after a second procedure, ${ }^{2}$ Germano et al $63 \%,{ }^{5}$ Gonzalez-Martinez $38 \%,{ }^{6}$ and Salanova $57 \%{ }^{7}$

With recent advances in neuroimaging and more frequent use of noninvasive video-electroencephalography (VEEG) monitoring, a more comprehensive and accurate evaluation of the epileptogenic zone should be accomplished before the first epilepsy surgery. In patients with intractable partial epilepsy there are many possible factors, alone or in combination, that could be related to the failure of resection. Some of the factors could be related to acquired and genetic predisposition to continuous seizures. 


\section{Specific case to consider}

We present a 27-year-old, right-handed male with no previous history of febrile seizures, head trauma, central nervous system infections or family history of seizures.

Since the age of 5 , the patient had complex partial seizures with and without secondary generalization plus occasional grand mal seizures. The seizures were not preceded by an aura and the patient had oral and bimanual automatisms, bipedal automatisms and loss of awareness during the seizures with no postictal confusion. This patient had a high frequency of seizures (10-15 seizures per day) since his epilepsy began. Over the years he failed to the following medications: topiramate, carbamazepine, levetiracetam and valproic acid.

This patient had the first investigation for epilepsy surgery when he was 17 years old. Before surgery he was taking the combination of valproic acid and carbamazepine with adequate doses, and adequate therapeutic levels with no response. In pre-operative investigation, scalp EEG showed his seizures originated over the right frontal area. The patient had intracranial recording with electrodes covering the orbito-frontal area, the frontal convexity and the fronto-polar area, which supported the previous localization.

The patient had a small resection of the fronto-polar area (Figure 1). He was rendered seizure free for 6 months and the medications were stopped. Pathology was consistent with cortical dysplasia.

At the end of the first year after surgery the patient's seizures restarted with the same frequency as before the intervention. The patient was retreated with the same medications used before surgery with no response and in the following years he failed to levetiracetam and topiramate. Also the patient was implanted with a vagal nerve stimulator when he was 20 years old, with no response.

The patient had a second investigation when he was 22 years old. The scalp EEG pointed to the seizure onset being over the right frontal area. Two ictal single photon emission computed tomography (SPECT) scans were taken showing an onset over the right orbito-frontal area. A decision was made to perform a right orbito-frontal resection without the necessity of intracranial recording (Figure 2). The pathology was consistent with cortical dysplasia. The resection was performed and the patient rendered seizure free for 6 months and then the medications were stopped.

At the end of the first year after surgery, patient's seizures restarted with the same frequency as prior to surgery. Before the second procedure patient was on the combination of topiramate and levetiracetam. These medications were restarted after the surgery with no success. Over the years

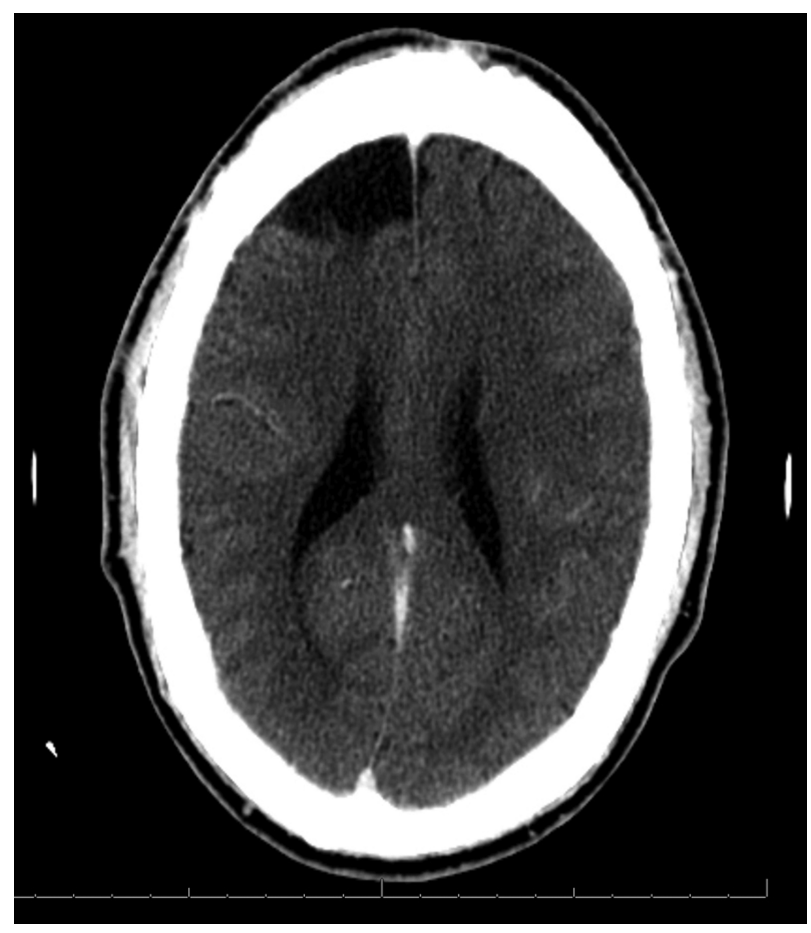

Figure I Axial CT scan showing the first resection in this patient over the superior aspect of the right frontal convexity.

more medications were added due to the high frequency of seizures and before being assessed in our center he was on a combination of 4 medications, clobazam $10 \mathrm{mg}$ twice daily, lamotrigine $250 \mathrm{mg}$ twice daily, phenytoin $400 \mathrm{mg}$ per day, and oxcarbazepine $600 \mathrm{mg}$ twice daily. A telemetry investigation was done recording 40 seizures with a potential onset over the right frontal vs temporal area (see Figure 3A), with very rapid secondary generalization.

Interictally, the EEG also showed independent right frontal and temporal spikes (Figure 3B) and during sleep the EEG showed a generalized polyspike wave (Figure 3C). An intracranial investigation was performed in this patient covering the remaining areas of the right frontal lobe and the temporal area (Figure 3D). The seizures have a simultaneous onset over the right frontal convexity and the neocortical aspect of the temporal area (Figure 3C).

A right frontal resection was performed sparing the motor strip and a standard temporal lobectomy was also performed. The patient had some postoperative seizures in the first 2 days characterized by jerks in the face and arm. These disappeared after 1 week and were attributed to inflammation related with the intracranial procedure. These seizures were treated with valproic acid IV, a daily dose of $20 \mathrm{mg}$ per $\mathrm{kg}$ for 7 days. The patient did not have any modification in the anti-epileptic drugs and was rendered seizure free after 1 week. 


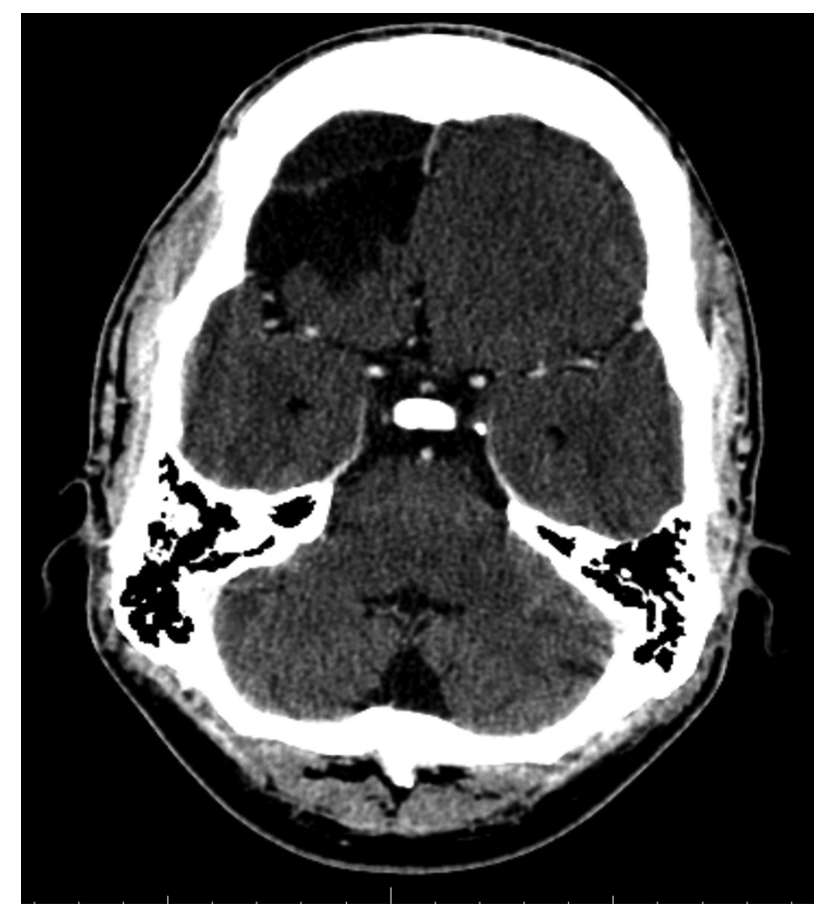

Figure $\mathbf{2}$ The second resection, involving the right orbito-frontal region.
The patient remained seizure free for 2 years with no modifications in the medications. No cognitive testing was done before surgery because of the high frequency of seizures and no significant cognitive decline was seen after surgery. The patient was able to get a driver licence after 1 year and started working as a clerk.

\section{Discussion}

This patient had 3 surgical interventions before he became seizure free. This is a very illustrative case of refractory epilepsy and shows many aspects common to all patients requiring reoperation after epilepsy surgery.

Potential reasons for surgery failure are as follows:

1. Extent of the first resection. Some centers have reported that the extent of the resection is an important factor for seizure recurrence. Holmes et al described 21 patients who had previous epilepsy surgery with recurrence of seizures, most of whom required extension of previous resections. ${ }^{8}$ Currently the tendency in all epilepsy centers is to perform more selective resections or less aggressive

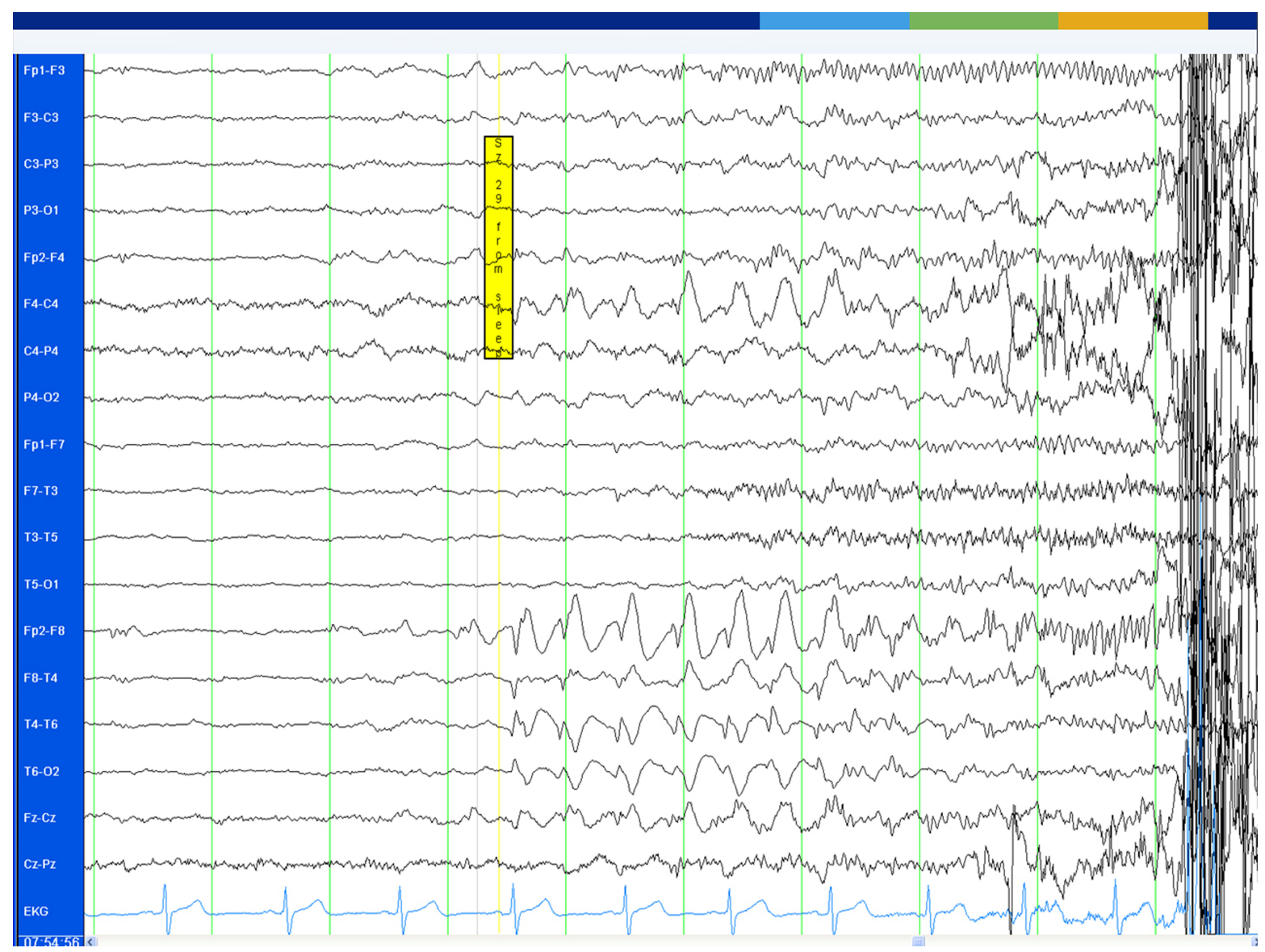

Figure 3A The onset of one of the seizures recorded with the scalp EEG. The EEG trace shows a simultaneous onset in the right temporal and frontal regions, involving the electrodes F4, Fp2, F8, T4 and some spread to T6. 


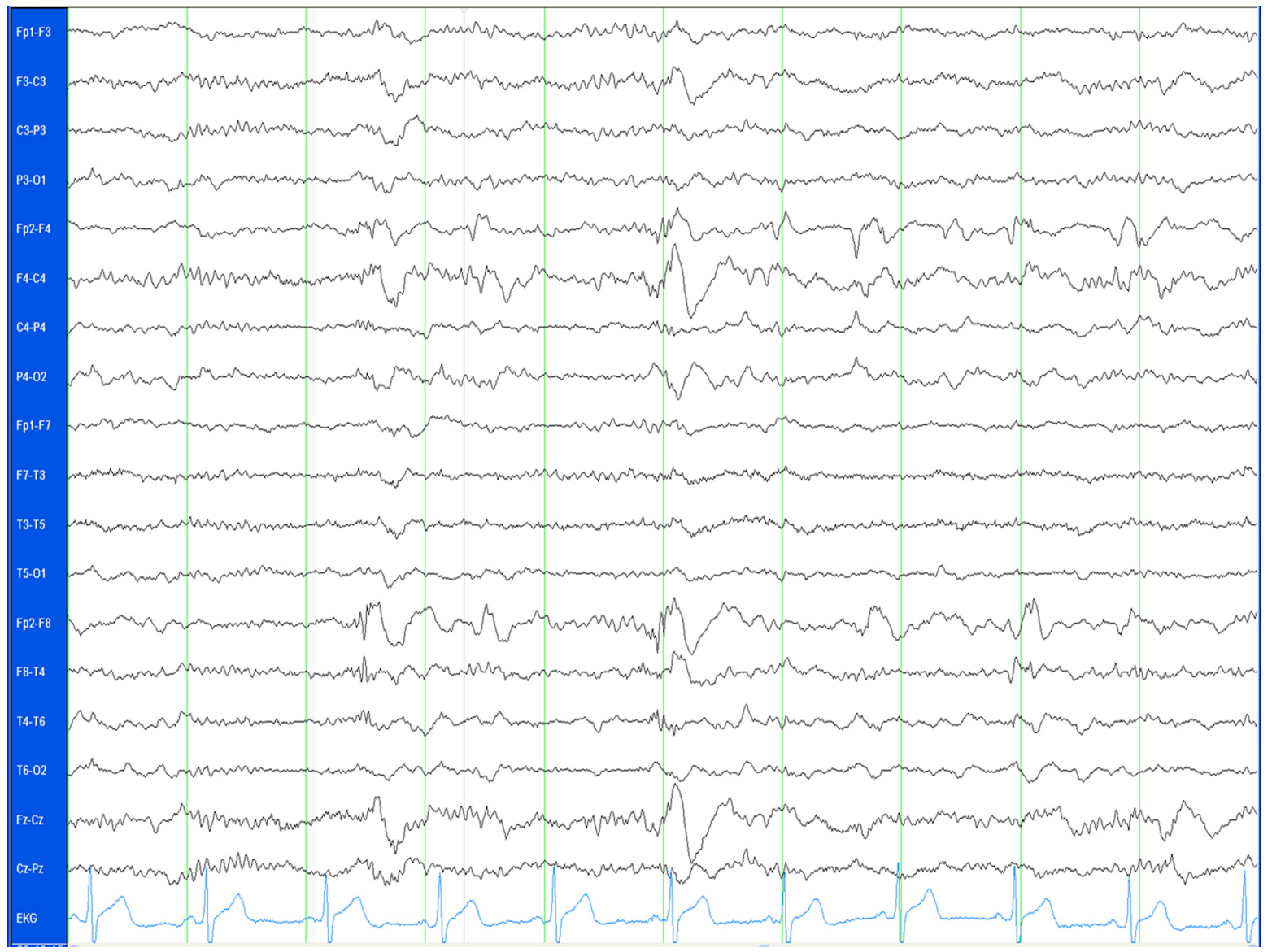

Figure 3B An EEG time segment displaying independent right frontal and right temporal spikes.

procedures in order to spare other mental functions in patients. This patient finished with a very aggressive resection in order to control the seizures without a significant cognitive decline, supporting the possibility to perform aggressive procedures in selected patients.

2. Incorrect mapping or erroneous identification of the epileptogenic area. The second aspect that could be discussed in this patient is the possibility that the epileptogenic area was not correctly mapped in the first intracranial investigation. One of the disadvantages of intracranial recording includes limited cortical sampling with consequent sampling error. ${ }^{9}$ This aspect is important in our case because the first investigation only covered the frontal area. The third investigation was more extensive, also covering the temporal area that showed a simultaneous onset with the frontal area.

3. Normal MRI before surgery. In his series of reoperations, Hennessy et al pointed out that some patients with reoperations had initial normal MRI and then in a second evaluation some abnormalities were identified..$^{10}$ The nonlesional status is a well recognized factor that predisposes to recurrence of seizures after epilepsy surgery. ${ }^{11}$ Our patient had several MRIs over the years with no findings. This fact was probably an independent risk factor for reoperation.

4. Interpretation of test. Another aspect in this patient is the ictal SPECT. This case showed that decisions based only on one test such as the SPECT probably are not adequate. Currently the evidence does not support the use of PET and SPECT in isolation to make surgical decisions. It has been shown that ictal SPECT is most useful in patients with extratemporal focal epilepsy to guide the placement of intracranial electrodes at the possible ictal onset zones. ${ }^{12,13}$ The PET scan is more of a lateralizing value, especially in temporal lobe epilepsy compared to extratemporal epilepsy. ${ }^{14}$

5. Pathological diagnosis. The pathological diagnosis in this patient was consistent with cortical dysplasia. This is an important consideration because it could explain in part the reoperations. Gonzalez-Martinez reported 57 patients who had temporal and extratemporal resections, and showed that patients with confirmed cortical dysplasia or mesial temporal sclerosis in temporal cases had worse outcome after reoperation. ${ }^{6}$ 


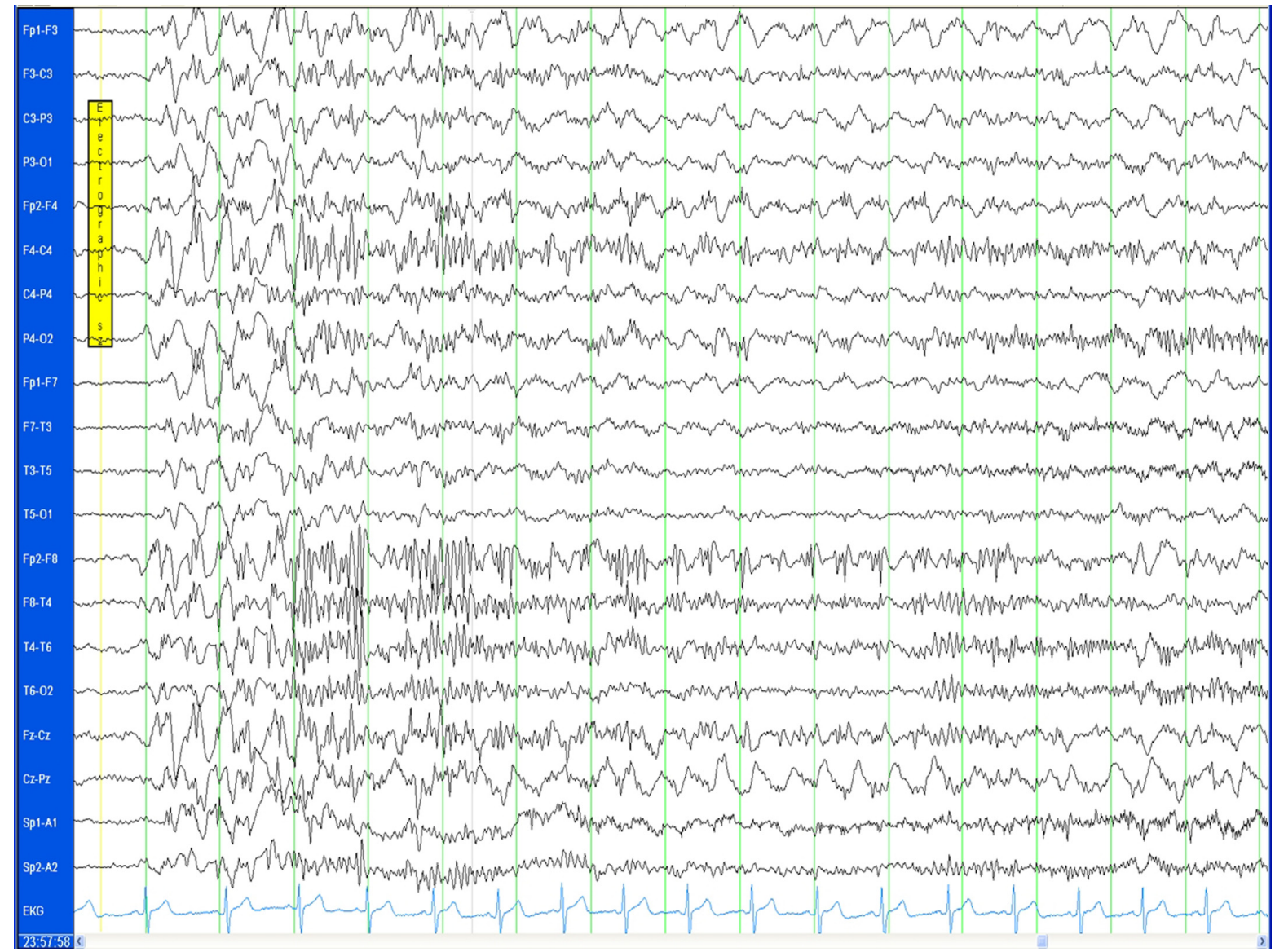

Figure 3C An EEG time segment during sleep showing generalized polyspike wave.

6. Duration of epilepsy. Siegel et al described in a series of 64 patients with reoperations. ${ }^{15}$ In this study patients with duration of epilepsy greater than 5 years had more possibilities of reoperation. This risk factor was present in our study, because our patient began to experience seizures at the age of 5 years and had a history of epilepsy of 22 years.

7. Multifocal seizure onset and candidacy for surgery. In a series of reoperated patients, a common reason of failure and reoperation is a previously demonstrated multifocal seizure onset. ${ }^{6}$ In the majority of these cases the surgery is used for palliative reasons. Salanova et al described some cases of reoperations where the resection was initially frontal and then the second resection involved temporal structures, suggesting that multifocal seizure onset is an important reason for reoperation. ${ }^{16}$

8. Development of new epileptogenic foci. Awad et al described some patients with new distant epileptogenic areas, suggesting the development of new epileptogenic foci after the first resection. ${ }^{2}$ In our case the involvement of the temporal area could be a new epileptogenic area but could possibly be an epileptogenic area that was not discovered initially.

9. Coexistence of generalized epilepsy. Hennessy et al described the neurophysiological findings of a group of patients with reoperations. Few patients had generalized epileptiform discharges. ${ }^{10}$ The possibility that some patients with reoperations can have coexistence of primary generalized epilepsy in addition to focal epilepsy is an important consideration. The fact that our patient had both types of epilepsy could be an additional risk factor for reoperation.

10. History of encephalitis or head injury. Recent series have described that previous head injury or encephalitis could be a strong predictor of epilepsy surgery failure. ${ }^{7,17}$ The potential consequence of these types of events in life is a more widespread involvement in the brain with the possibility of multiple epileptogenic foci. Our patient did not have any of these risk factors that could explain the recurrence.

11. Incomplete resections. The most common reported situation in reoperated cases is the presence of residual 


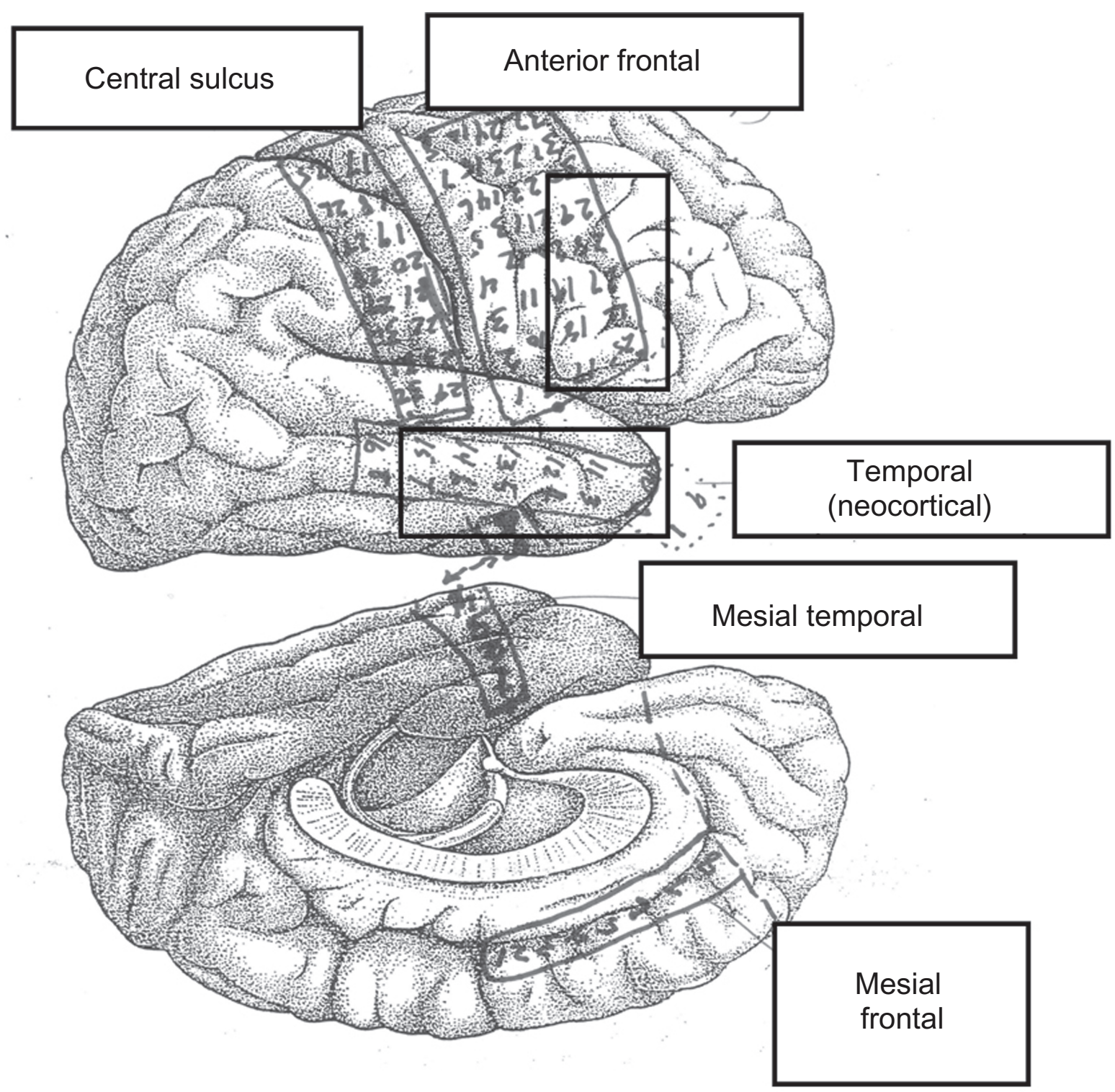

Figure 3D The placement of electrodes in this patient. The seizure onset for the 40 seizures is displayed in the two boxes, corresponding to the right frontal convexity and the neocortical aspect of the temporal area.

mesial structures after temporal resections. Germano et al reported 40 patients with temporal epilepsy that had a second resection. ${ }^{5}$ The imaging studies after surgery show that mesial structures were remaining in these 40 patients. The prognosis of patients after reoperation in this study was favorable, with $60 \%$ of patients rendered seizure free after surgery.

12. Lack of febrile seizures. Salanova et al compared 41 patients with seizure recurrence with 170 patients that were rendered seizure free after temporal resections. ${ }^{7}$ They found that the lack of febrile seizures was an indicator for reoperation. Therefore, lack of previous febrile seizures in our patient could be an indicator for reoperation.
13. Scarring after surgery. Schwartz et al described some patients who had reoperations where the potential reason was secondary scarring after epilepsy surgery, in the vicinity of the previous resected epileptogenic area. ${ }^{4}$ In our patient this factor could have been relevant. The first two surgeries may have triggered secondary scarring in the frontal area, increasing the risk of recurrence.

14. Genetic predisposition. We do not have enough information for a definite conclusion, but potentially a genetic predisposition could contribute to the risk of reoperations. The genetic predisposition to have seizures occurs more frequently in patients with generalized epilepsy, although some studies have suggested an increased genetic predisposition in some patients with partial 
epilepsy, potentially explaining the coexistence of generalized and focal epilepsy in the same patient, resulting in intractable epilepsy and potentially reoperations. ${ }^{18}$

\section{Conclusions}

Resective epilepsy surgery is the most effective treatment for patients with pharmacoresistant, localization-related epilepsy, and surgery is the intervention that is most likely to render patients free from seizures.

The frequency of reoperation reported in the literature is variable, ranging from $3 \%$ to $14 \%$.

When surgery fails, the correct understanding of why seizures occur, identifying patients who are at risk, and how to manage adverse outcomes when they do occur, is one of the most important endeavors facing epileptologists today.

Multiple factors are related to reoperations. Some of these are acquired characteristics of the patients, others are related to pitfalls in the investigation of patients, genetic predisposition, imaging or pathologic findings, and development of new epileptogenic foci.

Reoperation may be an appropriate alternative form of treatment for selected patients with intractable partial epilepsy who fail to respond to initial surgery.

\section{Disclosure}

Dr Téllez-Zenteno receives grants from the Royal University Hospital Foundation in Saskatoon and the University of Saskatchewan.

\section{References}

1. Téllez-Zenteno JF, Dhar R, Wiebe S. Long-term seizure outcomes following epilepsy surgery: a systematic review and meta-analysis. Brain. 2005;128:1188-1198.

2. Awad IA, Nayel MH, Luders H. Second operation after the failure of previous resection for epilepsy. Neurosurgery. 1991;28:510-518.
3. Awad IA. Reoperation for intractable seizures. Clin Neurosurg. 1992;39:125-139.

4. Schwartz TH, Spencer DD. Strategies for reoperation after comprehensive epilepsy surgery. J Neurosurg. 2001;95:615-623.

5. Germano IM, Poulin N, Olivier A. Reoperation for recurrent temporal lobe epilepsy. J Neurosurg. 1994;81:31-36.

6. Gonzalez-Martinez JA, Srikijvilaikul T, Nair D, Bingaman WE. Longterm seizure outcome in reoperation after failure of epilepsy surgery. Neurosurgery. 2007;60:873-880.

7. Salanova V, Markand O, Worth R. Temporal lobe epilepsy analysis of failures and the role of reoperation. Acta Neurol Scand. 2005;111:126-133.

8. Holmes MD, Wilensky AJ, Ojemann LM, Ojemann GA. Predicting outcome following reoperation for medically intractable epilepsy. Seizure. 1999;8:103-106.

9. Dubeau F, McLachlan RS. Invasive electrographic recording techniques in temporal lobe epilepsy. Can J Neurol Sci. 2000;27 Suppl 1: S29-S34.

10. Hennessy MJ, Elwes RD, Binnie CD, Polkey CE. Failed surgery for epilepsy. A study of persistence and recurrence of seizures following temporal resection. Brain. 2000;123 Pt 12:2445-2466.

11. Berkovic SF, McIntosh AM, Kalnins RM, et al. Preoperative MRI predicts outcome of temporal lobectomy: an actuarial analysis. Neurology. 1995;45:1358-1363.

12. O’Brien TJ, So EL, Mullan BP, et al. Subtraction peri-ictal SPECT is predictive of extratemporal epilepsy surgery outcome. Neurology. 2000;55:1668-1677.

13. O'Brien TJ, So EL, Cascino GD, et al. Subtraction SPECT coregistered to MRI in focal malformations of cortical development: localization of the epileptogenic zone in epilepsy surgery candidates. Epilepsia. 2004;45:367-376.

14. Lee SK, Lee SY, Kim KK, Hong KS, Lee DS, Chung CK. Surgical outcome and prognostic factors of cryptogenic neocortical epilepsy. Ann Neurol. 2005;58:525-532.

15. Siegel AM, Cascino GD, Meyer FB, et al. Resective reoperation for failed epilepsy surgery: seizure outcome in 64 patients. Neurology. 2004;63:2298-2302.

16. Salanova V, Quesney LF, Rasmussen T, Andermann F, Olivier A. Reevaluation of surgical failures and the role of reoperation in 39 patients with frontal lobe epilepsy. Epilepsia. 1994;35:70-80.

17. Ramos E, Benbadis S, Vale FL. Failure of temporal lobe resection for epilepsy in patients with mesial temporal sclerosis: results and treatment options. J Neurosurg. 2009;110:1127-1134.

18. bou-Khalil B, Krei L, Lazenby B, Harris PA, Haines JL, Hedera P. Familial genetic predisposition, epilepsy localization and antecedent febrile seizures. Epilepsy Res. 2007;73:104-110.
Neuropsychiatric Disease and Treatment

\section{Publish your work in this journal}

Neuropsychiatric Disease and Treatment is an international, peerreviewed journal of clinical therapeutics and pharmacology focusing on concise rapid reporting of clinical or pre-clinical studies on a range of neuropsychiatric and neurological disorders. This journal is indexed on PubMed Central, the 'PsycINFO' database and CAS, and is the official

\section{Dovepress}

journal of The International Neuropsychiatric Association (INA). The manuscript management system is completely online and includes a very quick and fair peer-review system, which is all easy to use. Visit http://www.dovepress.com/testimonials.php to read real quotes from published authors. 